\title{
A VIVÊNCIA DA PESSOA COM DIABETES APÓS ATENDIMENTO DE URGÊNCIA*
}

\author{
Simone Cristina Baggio', Jocimara Costa Mazzola², Sonia Silva Marcon ${ }^{3}$
}

RESUMO: O objetivo deste estudo descritivo-exploratório com abordagem qualitativa foi compreender os fatores intervenientes no tratamento do diabetes mellitus que levam pessoas com esse diagnóstico a necessitar de atendimento de saúde de urgência. O estudo foi realizado em setembro de 2009 com 18 pessoas diabéticas; os dados foram coletados por meio de entrevista semiestruturada e submetidos à análise de conteúdo. Os resultados mostram que, entre os cuidados cotidianos para o controle da doença, o domínio da alimentação e o uso correto das medicações são os mais valorizados e que a dieta com restrições é a principal dificuldade enfrentada. Concluímos que os indivíduos estudados necessitam de orientações para o aprimoramento de habilidades, sendo imprescindível que as equipes de saúde conheçam a realidade individual e que estejam capacitadas e volvidas à sistematização da assistência visando mudanças de hábitos de vida e contribuindo com o controle da síndrome metabólica.

PALAVRAS-CHAVE: Diabetes mellitus; Conhecimento; Educação em saúde.

\section{THE EXPERIENCE OF PEOPLE WITH DIABETES AFTER ATTENDING ACCIDENT AND EMERGENCY}

\begin{abstract}
The objective of this descriptive-exploratory study with a qualitative approach was to understand the intervening factors in the treatment of diabetes mellitus which lead people with this diagnosis to need to attend Accident and Emergency units. The study was carried out in September 2009 with 18 diabetic people; the data were collected via semi-structured interviews and submitted to content analysis. The results showed that among daily measures taken to control the illness, control of food and the correct use of medicines are the most valued, and that the restrictive diet is the main difficulty met. It was concluded that the individuals studied need advice to improve their abilities, it being vital that health teams are familiar with individual realities and that they are enabled and oriented to systematization of help, aiming at changing life habits and contributing to the control of the metabolic syndrome.
\end{abstract}

KEYWORDS: Diabetes mellitus; Knowledge; Health education.

\section{LA VIVENCIA DE LA PERSONA CON DIABETES DESPUES DE ATENDIMIENTO DE URGENCIA}

RESUMEN: El objetivo de este estudio descriptivo-exploratorio con abordaje cualitativo fue comprender los factores que intervienen en el tratamiento del diabetes mellitus los cuales llevan personas con ese diagnóstico a necesitar de atendimiento de salud de urgencia. El estudio fue realizado en septiembre de 2009 con 18 personas diabéticas; los datos fueron recogidos por medio de entrevista semiestructurada y sometidos al análisis de contenido. Los resultados muetran que, entre los cuidados cotidianos para el control de la enfermedad, el dominio de la alimentación y el uso correcto de las medicaciones son los más valorados y que la dieta con restricciones es la principal dificultad afrontada. Se concluyó que los individuos estudiados necesitan orientaciones para mejorar habilidades, siendo imprescindible que los equipos de salud conozcan la realidad individual y que sean capacitadas y habilitadas a la sistematización de la asistencia con el objetivo de cambiar hábitos de vida y contribuyendo con el control del síndrome metabólico.

PALABRAS CLAVE: Diabetes mellitus; Conocimiento; Educación en salud.

\footnotetext{
*Pesquisa desenvolvida na disciplina Tópicos Avançados: Cuidado à Saúde nos Diferentes Ciclos da Vida do Mestrado em Enfermagem da Universidade Estadual de Maringá - UEM.

${ }^{1}$ Enfermeira. Mestranda em Enfermagem na UEM. Professora do Departamento de Enfermagem da Universidade de Paranavaí.

${ }^{2}$ Enfermeira. Mestranda em Enfermagem na UEM.

${ }^{3}$ Enfermeira. Doutora em Filosofia da Enfermagem. Professora do Departamento e do Programa de Mestrado em Enfermagem da UEM.
}

Autor correspondente:

Simone Cristina Baggio

Universidade Estadual de Maringá

R. Miljutin Cogei, 165 - 87701-090 - Paranavaí-PR-Brasil

Recebido: 09/02/11

E-mail: simone_baggio@hotmail.com

Aprovado:09/05/11 


\section{INTRODUÇÃO}

O diabetes mellitus (DM) é considerado um importante problema de saúde pública na atualidade devido aos elevados índices de morbimortalidade e o impacto negativo da doença sobre o doente, a família e a sociedade, em função de suas complicações, podendo levar à invalidez precoce. A enfermidade tem impacto na qualidade de vida e sobrevida dos doentes, além dos prejuízos econômicos causados pelo alto custo do tratamento e frequentes internações ${ }^{(1)}$.

Os índices crescentes da doença podem estar relacionados com o aumento da expectativa de vida e a maior sobrevida da pessoa com DM, em decorrência dos avanços tecnológicos, maior taxa de urbanização e industrialização, hábitos alimentares inadequados, sedentarismo, obesidade e estresse ${ }^{(2)}$.

Diante disso, o diabetes é considerado uma condição crônica de saúde que demanda do doente atenção e conhecimento para melhor condução do seu complexo tratamento. Este objetiva, não somente manter os níveis glicêmicos normais, mas também o controle dos fatores de risco que concorrem para a morbimortalidade da doença ${ }^{(2)}$.

Por esta razão, as mudanças no estilo de vida, sobretudo em relação à prática alimentar e de atividade física, constituem a base para o tratamento e o controle da doença ${ }^{(3)}$. O controle metabólico rigoroso, combinado às medidas preventivas e curativas, relativamente simples, são eficazes para a prevenção ou retardamento de complicações crônicas do DM, resultando em melhor qualidade de vida ao indivíduo diabético ${ }^{(4)}$.

Assim o envolvimento da família no controle do diabetes se faz importante, pois pode influenciar positivamente os hábitos de vida do doente crônico. Cabe salientar que o bem viver do indivíduo diabético, assim como de sua família, está fundamentado na prática do cuidado que, por sua vez, é permeado por crenças, valores e costumes ${ }^{(1)}$.

Portanto, é imprescindível que os profissionais da saúde, no âmbito primário e secundário da assistência, estejam capacitados e desenvolvam ações de cuidado em saúde visando maior envolvimento do doente e participação da família no controle da doença crônica, sendo este um desafio para as equipes de saúde. Diante do exposto, o objetivo do estudo foi compreender os fatores intervenientes do DM que levam as pessoas a necessitar de atendimento de saúde de urgência/emergência.

\section{METODOLOGIA}

Trata-se de um estudo descritivo/exploratório com abordagem qualitativa. Participaram do estudo 18 indivíduos diabéticos que buscaram atendimento de urgência/emergência no mês de agosto de 2009, por descompensação dos níveis glicêmicos, no prontoatendimento municipal de Maringá (8 indivíduos) e no pronto-atendimento municipal de Paranavaí (10 indivíduos), ambas cidades do Estado do Paraná.

Para identificação dos indivíduos do estudo foram analisados os prontuários médicos desse período e, mediante as anotações médicas e de enfermagem, identificou-se as principais situações que resultaram na busca pelo atendimento de caráter de urgência e/ ou emergência (hiperglicemia e hipoglicemia). Nesse momento, foram relacionados os pacientes acometidos, e registro do nome completo, endereço e telefone de contato.

Em seguida, os sujeitos foram contatados por telefone e solicitados a participar no estudo e então realizou-se uma visita domiciliar em dia e horário conveniente a estes; após o consentimento formal, as entrevistas foram gravadas. A entrevista semiestruturada foi realizada utilizado-se um roteiro constituído de duas partes: a primeira contendo 12 questões referentes à identificação do indivíduo e da família e a segunda com 15 questões abertas que abordavam os hábitos e estilo de vida, conhecimento e percepção sobre a doença, a procura pelo atendimento de saúde na situação de urgência/emergência e cuidados adotados para o controle da doença.

As falas das entrevistas foram transcritas na íntegra. Para a análise dos dados foi utilizado o referencial metodológico, o qual pode ser entendido como um conjunto de técnicas de análise das comunicações visando obter, por procedimentos sistemáticos e objetivos de descrição do conteúdo das mensagens, indicadores que permitam a inferência de conhecimentos relativos às condições de produção/recepção destas mensagens ${ }^{(5)}$.

Para tanto, iniciou-se a análise dos dados brutos, procedentes da transcrição das entrevistas, por meio de leituras flutuantes e, subsequentemente, foram feitas inúmeras leituras detalhadas, o que oportunizou a identificação dos componentes para as interpretações e o desprendimento das variáveis de inferência. Posteriormente, foi possível obter recortes das unidades de registro denominadas, até então, sob um título genérico. Em seguida, discutiram-se os dados obtidos tomando por base as publicações científicas sobre o tema. 
O desenvolvimento do estudo atendeu as exigências da Resolução 196/96 do Conselho Nacional de Saúde com aprovação do projeto pelo Comitê Permanente de Ética em Pesquisa Envolvendo Seres Humanos da Universidade Estadual de Maringá sob parecer n. 632/2009. Todos os participantes do estudo assinaram o Termo de Consentimento Livre e Esclarecido; para garantir o anonimato, os entrevistados foram identificadas pelas letras iniciais do nome, seguidas do sexo e idade.

\section{RESULTADOS}

Considerando os depoimentos dos participantes do estudo sobre o conhecimento da doença e os fatores intervenientes ao seu controle, emergiram três categorias empíricas: "definindo o diabetes", "dificuldades e facilidades no controle da doença" e "fatores intervenientes da descompensação metabólica".

\section{Definindo o diabetes}

Esta categoria engloba o significado do diabetes mellitus para os portadores; com isso, percebeu-se que alguns fazem referência à doença como limitante e grave:

É uma doença nojenta [...] tem que ter outra vida, é tudo diferente. (NSS,F,56)

\section{É uma doença muito perigosa [...]. (FJA,F,63)}

É a pior coisa que tem no corpo de uma pessoa. (MIPS,F,58)

É alguma coisa que atrapalha, mas temos que manter a coragem para viver [...]. (MRS,M,75)

O DM é definido com conhecimento alicerçado no senso comum, elaborado pelo ser humano desde a antiguidade:

Diabetes é açúcar no sangue. (APB,F,61)

Em outros relatos, observa-se que a doença é associada às possíveis causas e consequências:

Idade, comer muito doce e ser gordo. (MRS,M,75)

Não evitar gordura, exagerar na comida, e comer muito açúcar. (IPT,F,80)

Uma pessoa com diabetes tem muitos problemas, pode perder a visão ou ter que cortar um dedo ou uma perna fora. (APB,F,61)

Isoladamente, percebe-se que o DM confere ao doente revolta e sentimentos de angústia e a não-aceitação de uma vida limitada e controlada, levando-o a definir a doença segundo os sentimentos experienciados:

O inferno da minha vida. Não aceito a doença. (IPT,F,80)

\section{Dificuldades e facilidades no controle da doença}

Os entrevistados explicitam as dificuldades cotidianas relacionadas com a alimentação para o controle da doença, em especial, com o consumo de alimentos ricos em carboidratos, glicídios e gorduras:

A dificuldade é só a boca. Não comer doce, gordura, é difícil controlar. (MIPS,F,58)

A dificuldade é que não pode comer as coisas boas, carne gorda, pão, massa, macarrão muito pouco, doce nem pensar. (JAMN,M,72)

O mais difícil é a mudança de vida. Tem que parar de sair, de beber e comer. (DASM,F,52)

Ainda associou-se o estado emocional como sendo um fator dificultador no controle do DM, com sintomas das alterações glicêmicas após um episódio de crise nervosa:

É difícil controlar quando fico nervoso, ai já sinto o mal-estar da diabete subindo. (GT,M,55)

É dificil por que sou muito nervosa. (IPT,F,80)

É importante destacar os fatores de promoção e apoio apontados pelos diabéticos para o controle da doença:

O que ajuda a controlar a diabete é não comprar coisas doces, massas e refrigerantes. (VJB,F,75)

Facilita controlar a doença usar pouca gordura para cozinhar e comer carnes magras. (ERA,F,67)

Ajuda a controlar a diabetes andar mais e comer menos e tomar mais chá caseiro, como chá da folha de insulina. (JCF,F,64) 
O que facilita eu controlar a minha diabete é por que sou calma, não como doce e sigo a dieta. (APB,F,61)

Diante das limitações, dificuldades e facilidades vivenciadas pelos diabéticos, buscou-se saber as ações diárias desenvolvidas para o controle da doença:

Faço a dieta não comendo muito e tomo os remédios no horário certo. (VJB,F,75)

Faço dieta na alimentação, evito comer massas e doces e estou fazendo caminhada. (MSCT,F,51)

Faço uso dos remédios, exame do dedo e dieta. (CSS,F,21)

Faço o exame do dedo duas vezes por dia, dependendo do resultado diminuo a minha comida. (MRS,M,75)

\section{Fatores intervenientes da descompensação meta- bólica}

Ao investigar junto aos indivíduos diabéticos o que determinou a procura pelo serviço de urgência/ emergência, constatou-se que esses fizeram referência a um conjunto de sintomas que permitem constatar a elevação glicêmica associando-a aos possíveis fatores que contribuíram para esta elevação, a exemplo:

Alimentação inadequada.

Suava frio, aumentou a vontade de comer e de fazer xixi, angústia. [...] porque não faço a dieta como tem que fazer. (MRS,M,75)

Dor de cabeça e no estômago, vômito, as veias do braço ficaram murchas e escuras [...] quando como muito arroz, macarrão e doce. (CSS,F,21)

Fator emocional.

Sentia muita sede, boca seca, muito suor frio. É muito ruim [...] acho que foi o emocional. Fiquei muito nervosa. (MSCT,F,51)

Dor nas pernas, sede a gente bebe muita água, vista escura [...] ela sobe mais quando fico nervosa. (MIPS,F,58)

Uso irregular dos medicamentos.

Dor nas pernas, muita sede, olhos embaçados e fazia muito xixi. [...] porque não tomo o remédio na hora certa. (GT,M,55)

Diante das adversidades vivenciadas, os diabéticos manifestaram necessidade de ajuda para se readaptar ao novo estilo de vida. Eles consideraram importante a assistência recebida do profissional de saúde, explicitando a valorização das informações e orientações recebidas de enfermeiros e médicos:

O médico da família ia na minha casa a cada 15 dias [...] serviu para eu saber como lidar com a doença. (MRS,M,75)

Médico e enfermeira em reuniões [...] aprendi a aplicar insulina e coisas que não sabia sobre a diabetes [...] foi importante para evitar comer coisas que não pode. (ERA,F,67)

Recebi informação do enfermeiro do posto. Foi muito bom, porque ele falou para não comer muito doce, massa [...]. Mudei o jeito de comer e quando as pessoas explicam pra gente é melhor, a gente aprende. (NS,F,64)

\section{DISCUSSÃO}

Partindo do pressuposto de que o conhecimento é ponto fundamental para o controle e tratamento do $\mathrm{DM}$, podemos inferir que o grupo em estudo carece de ações de educação em saúde que favoreçam suas competências para o automanejo e controle da doença.

$\mathrm{O}$ investimento na educação da pessoa com diabetes, além de beneficiar o doente, pois o ajuda no controle da doença ${ }^{(6)}$, em especial no controle dos níveis glicêmicos, também parece conferir outros benefícios, como a redução significativa do risco de complicações e de gastos em saúde ${ }^{(7)}$.

Diante da condição de doente crônico, o indivíduo precisa aprender determinadas competências que lhe possibilite lidar no dia-a-dia com os sintomas e limitações. Para que isso se concretize, é importante que tenha o conhecimento correto sobre a sua saúde e doença, de forma que seja capaz de resolver os problemas advindos da doença e apto para implementar um comportamento saudável ${ }^{(7)}$.

Desta forma, identificou-se que para os diabéticos do estudo não havia distinção entre os cuidados praticados e os fatores facilitadores do controle glicêmico. Assim, quando os indivíduos diabéticos se deparam 
com a doença, as rotinas são modificadas e/ou adaptadas em prol de cuidados inerentes à doença. Dentre essas readaptações, eles pontuam cuidados importantes como facilitadores do controle glicêmico. O domínio sobre a alimentação e o uso correto dos remédios são considerados os principais cuidados para o controle e tratamento da doença, seguidos do monitoramento da glicemia capilar e prática de atividade física.

A restrição alimentar é uma invariável no cotidiano da pessoa diabética e a reeducação alimentar é fator definitivo na sua vida. Nesse sentido, a educação é essencial para a compreensão da necessidade de repensar o planejamento alimentar ${ }^{(8)}$.

Torna-se imprescindível que o diabético observe a característica dos alimentos no momento da refeição, devendo ter um cuidado criterioso para realizar uma refeição balanceada e, ao mesmo tempo, respeitando as restrições dietéticas impostas pelo $\mathrm{DM}^{(4)}$.

Estudo realizado com indivíduos diabéticos internados revelou que um dos maiores déficits de autocuidado é o nutricional, sendo que a preincipal dificuldade apontada pelos participantes não foi diminuir a gordura e, sim, limitar o consumo de alimentos com açúcar ${ }^{(9)}$.

O uso criterioso da medicação foi apontado como outro fator fundamental para o alcance do controle metabólico, o que reforça os achados de um estudo realizado com 55 diabéticos no qual o conhecimento e a preocupação acerca da medicação podem contribuir com o estado de saúde da pessoa e, assim, constituir em acréscimo significativo ao controle metabólico e à prevenção de complicações ${ }^{(10)}$.

O uso correto da medicação é um dos pilares no tratamento do DM, porém, ele faz parte de um conjunto de observações diárias para o alcance da meta que é o controle metabólico.

A automonitorização da glicemia capilar foi outro cuidado relatado pelos participantes do estudo e considerada um importante recurso para a determinação dos índices de glicemia pré e pós-prandial. Também se constitui num valioso recurso educativo para a pessoa, pois em determinadas situações, em que há infração alimentar ou omissão de uma refeição, os valores vão indicar hiperglicemia ou hipoglicemia permitindo a identificação precoce da alteração e a tomada de condutas imediatas para o controle ${ }^{(12)}$.

A prática da atividade física, por sua vez, é um dos pilares do tratamento do DM e, no entanto, foi referida por apenas um dos entrevistados, o que denota a dificuldade de adesão a esta prática por este grupo de indivíduos. De certo modo, o fato é compreensível, pois envolve mudança de hábitos pessoais, alteração no cotidiano, motivação, valores, crenças e autoestima, além de estímulo por parte dos profissionais de saúde.

Um outro estudo enfatiza que iniciar e/ou implementar a prática de atividade física é um dos desafios do tratamento do paciente diabético e deve ser praticada com a orientação e o apoio de uma equipe multiprofissional $^{(8)}$.

Outra pesquisa mostrou que, ao longo do tempo de acompanhamento de um grupo de diabéticos que se adaptou à restrição alimentar e à prática de atividade física, muitos dos participantes obtiveram o sucesso do controle glicêmico por meio destes cuidados. Evidenciou-se, também, que muitos diabéticos deixaram de usar insulina depois da adoção desses cuidados ${ }^{(4)}$.

A literatura corrobora o fato, mostrando, por meio de estudo realizado com diabéticos sedentários inseridos em um programa de atividade física que, em três meses de exercícios programados, houve diminuição nos valores médios de hemoglobina glicosada, confirmando a importância da atividade física no controle metabólico ${ }^{(13)}$.

Os relatos revelam a consciência de cuidados simples para o controle da doença, os quais se assemelham com os fatores listados como os que atrapalham a manutenção dos níveis glicêmicos. Entretanto, é essencial o conhecimento demonstrado pelos diabéticos sobre os fatores que contribuem para o controle metabólico, sendo fundamental que o doente e a sua família compreendam a condição de doente crônico replanejando a vida diária com mudanças de hábitos e estilo de vida.

Outro aspecto que tem mostrado influência no controle da doença é o estado emocional, pois o diabetes exige um controle intenso para evitar complicações. A elaboração e aceitação da doença são pontos a serem desenvolvidos no doente para a obtenção de uma melhor qualidade de vida e do controle metabólico ${ }^{(14)}$. Assim, o controle do nível glicêmico ideal depende do equilíbrio emocional do indivíduo e, para isso, é fundamental o apoio da família ao doente ${ }^{(14)}$.

Estudo desenvolvido com um grupo de diabéticos do Município de Ijuí, no Rio Grande do Sul, mostrou que alguns entrevistados fizeram referência a determinadas situações de estresse e as consideraram colaboradoras para a alteração dos níveis glicêmicos. Ao passar por tais momentos, eles perceberam que as taxas de glicemia se modificaram, deixando-os preocupados ${ }^{(15)}$.

Outro estudo salienta que é amplamente reconhecido que o estado emocional desempenha um papel importante ao longo do curso do diabetes, por ação 
do sistema nervoso simpático, havendo a mobilização das hormonas contrarreguladoras que inibem a ação da insulina ${ }^{(16)}$.

Os discursos são reveladores do quanto o DM é percebido como uma doença ameaçadora, pois a principal causa pela procura do atendimento de saúde foi a hiperglicemia. Essa, por sua vez, em todos os casos foi acompanhada de manifestações clínicas diversas. É possível inferir que o reconhecimento dos sinais e sintomas da hiperglicemia constitui um fator protetor da vida. Entretanto, por se tratar de uma doença multifatorial, percebe-se que a descompensação metabólica tem igualmente uma particularidade multifatorial, abrangendo fatores relacionados não somente com os hábitos e estilo de vida, mas também com fatores emocionais.

Portanto, conviver com uma doença crônica foge da normalidade e esta nova realidade requer do doente um novo aprendizado. Este necessita desenvolver competências para lidar com a doença que compreende modificar hábitos, sendo esta uma tarefa árdua, porém, extremamente importante para o controle glicêmico.

$\mathrm{O}$ atendimento à saúde de um indivíduo com doença crônica deve abranger uma relação de afeto, respeito, capacitação, dedicação e comprometimento dos profissionais envolvidos em seu tratamento. Desta forma, o profissional de saúde deve buscar como foco fundamental da assistência o indivíduo e as suas necessidades em relação à doença, atuando como um agente educador, facilitador e também mobilizador, para melhorar a condição de vida das pessoas.

A educação em diabetes é o processo de ensinar os doentes a administrar a sua vida com a doença e o automanejo da mesma. Nessa direção, cabe à equipe multiprofissional, além de disponibilizar ao doente as informações sobre a sua doença, acompanhá-lo visando auxiliar na tomada de decisões, diante das inúmeras situações que o diabetes pode causar. Portanto, a equipe multiprofissional deve oferecer aos diabéticos os meios para o autocuidado ${ }^{(17)}$.

\section{CONSIDERAÇÕES FINAIS}

Os resultados deste estudo mostram que alguns diabéticos definem a doença com conhecimento alicerçado no senso comum, enquanto outros a associam com as causas, consequências e sentimentos negativos vivenciados.

Os participantes listaram fatores dificultadores e facilitadores no controle metabólico e cuidados importantes que devem ser adotados para o controle da doença. Porém, observou-se que não há distinção, para os pesquisados, entre fatores facilitadores para a manutenção dos níveis glicêmicos e cuidados praticados e entre os cuidados citados. Isso significa que, para o grupo estudado, as principais dificuldades são o replanejamento alimentar e a adoção e manutenção das restrições na dieta.

Outro aspecto importante do estudo é a valorização que os diabéticos conferiram à assistência recebida dos profissionais da saúde. Nesse sentido, faz-se necessário que os profissionais, especialmente os enfermeiros, identifiquem as necessidades do doente e, com ele, busquem ações para o controle da enfermidade.

Conclui-se que há a necessidade das equipes de saúde desenvolver estratégias de planejamento do cuidado ao indivíduo diabético, com maior envolvimento do mesmo e da sua família. Sendo assim, o profissional deve primeiramente buscar conhecer integralmente o doente e a sua família, entendendo as relações familiares e sociais que permeiam a vivência com a doença. Em seguida, devem promover ações em saúde, atuando de maneira a construir ou lapidar o conhecimento sobre o diabetes, contribuindo com o desenvolvimento de práticas domiciliares diárias saudáveis e seguras.

\section{REFERÊNCIAS}

1. Marcon SS, Lopes MCL, Fernandes J, Antunes CRM, Waidmann MAP. Famílias cuidadoras de pessoas com dependência: um estudo bibliográfico. Online Braz J Nurs. [Internet] 2006;5(1) [acesso em 08 abr 2009]. Disponível: http://www.uff.br/objnursing/index.php/ nursing/article/view/145/40>

2. Ministério da Saúde (BR). Vigitel 2007 - vigilância de fatores de risco e proteção para doenças crônicas por inquérito telefônico. Brasília: Ministério da Saúde; 2008.

3. American Diabetes Association. Diagnosis and classification of diabetes mellitus. Diabetes Care. 2008;27(Suppl1):5-10.

4. Góes APP, Vieira MRR, Liberatore Júnior RDR. Diabetes mellitus tipo I no contexto familiar e social. Rev Paul Pediatr. 2007;25(2):124-8.

5. Bardin L. Análise de conteúdo. $5^{\mathrm{a}}$ ed. rev. atual. Lisboa: Edições 70; 2009. 
6. Torres HC, Pace AE, Stradi MA. Análise sociodemográfica e clínica de indivíduos com diabetes tipo 2 e sua relação com o autocuidado. Cogitare Enferm. 2010;15(1):48-54.

7. Souza MRMGC, McIntyre T. Conhecimento do diabético sobre a doença e a repercussão no tratamento. Rev Bras Promoc Saúde. 2008;21(4):281-9.

8. Zanetti ML, Biagg MV, Santos MA, Péres DS, Teixeira CRS. O cuidado à pessoa diabética e as repercussões na família. Rev Bras Enferm. 2008;61(2):186-92.

9. Comiotto G, Martins, JJ. Promovendo o autocuidado do indivíduo diabético: da hospitalização ao domicílio. Arq Catarin Med. 2006;35(3):59-64.

10. Faria HTG, Zanetti ML, Santos MA, Teixeira CRS. Conhecimento sobre terapêutica medicamentosa em diabetes: um desafio na atenção à saúde. Acta Paul Enferm. 2009;2(5):612-7.

11. Gimenes HT, Zanetti ML, Otero LM, Teixeira CRS. O conhecimento do paciente diabético tipo 2 acerca dos antidiabéticos orais. Ciênc Cuid Saúde. 2006;5(3):317-25.

12. Franco VS, Zanetti ML, Teixeira CRS, Kusumota L. Automonitorização da glicemia capilar no domicílio. Ciênc Cuid Saúde. 2008;7(1):121-7.

13. Fechio JJ, Malerbi FEK. Adesão a um programa de atividade física em adultos portadores de diabetes. Arq Bras Endocrinol Metab. 2004;48(2):267-75.

14. Marcelino DB, Carvalho MDB. Reflexões sobre o diabetes tipo I e sua relação com o emocional. Psicol Reflex Crit. 2005;18(1):72-7.

15. Welfer M, Leite MT. Ser portador de diabetes tipo 2: cuidando-se para continuar vivendo. Sci Med. 2005;15(3):148-55.

16. Silva I, Ribeiro JP, Cardoso H. Dificuldade em perceber o lado positivo da vida? Stresse em doentes diabéticos com e sem complicações crônicas da doença. Análise Psicológica. 2004;3(XXII):597-605.

17. Zanetti ML, Otero LM, Peres DS, Santos MA, Guimarães FPM, Freitas MCF. Evolução do tratamento de pacientes diabéticos utilizando o protocolo staged diabetes management. Acta Paul Enferm. 2007;20(3):338-44. 